\title{
Clinical Observation on the Treatment of Chronic Subjective Dizziness by the Herbal-Scraping Duplex Method Based on Holographic Theory
}

\author{
Huanwen Luo, ${ }^{1}$ Caidan Liu, ${ }^{1}$ Ziwei Xiao, ${ }^{2}$ and Yana $\mathrm{He} \mathbb{D}^{3}$ \\ ${ }^{1}$ Department of Brain Disease, The First Hospital of Hunan University of Chinese Medicine, Changsha, Hunan 410000, China \\ ${ }^{2}$ Hunan University of Chinese Medicine, Changsha, Hunan 410000, China \\ ${ }^{3}$ Bidding Center of the First Hospital of Hunan University of Chinese Medicine, Changsha, \\ Hunan 410000, China \\ Correspondence should be addressed to Yana He; 909932870@qq.com
}

Received 9 December 2021; Accepted 31 December 2021; Published 24 January 2022

Academic Editor: Weiguo Li

Copyright (C) 2022 Huanwen Luo et al. This is an open access article distributed under the Creative Commons Attribution License, which permits unrestricted use, distribution, and reproduction in any medium, provided the original work is properly cited.

\begin{abstract}
Chronic subjective dizziness (CSD) is a chronic, subjective, nonrotational dizziness and instability caused by mental or physical factors, and patients generally have no existing vestibular system diseases. Clinically, antianxiety drugs are often used for symptomatic treatment with mediocre effects. This study observed the clinical efficacy of the herbal-scraping duplex method in the treatment of CSD based on the holographic theory. 180 patients with CSD were randomly and equally divided into group A $(n=60)$ who received fluoxetine hydrochloride, group B $(n=60)$ who received fluoxetine hydrochloride combined with holographic soup, and group C $(n=60)$ who received fluoxetine hydrochloride, holographic soup, and holographic scraping combined with five-element music. The effects of the 3 different treatment modalities on patients' vertigo symptoms, traditional Chinese medicine (TCM) symptoms, anxiety and depression status, sleep quality, and fatigue level were observed. The safety of medication and the recurrence after discontinuation were observed. The results showed that group $\mathrm{C}$ was better than group $\mathrm{B}$ and group $\mathrm{A}$ in terms of improvement in all of the above indicators, and group B was better than group A $(P<0.05)$. The total efficiency of Chinese and Western medicine was better in groups $\mathrm{C}$ and B than in group A $(P<0.05)$. No statistical difference was seen in the comparison of adverse reaction rates between the 3 groups $(P>0.05)$. After 1 month of drug discontinuation, the recurrence rates in groups $\mathrm{C}$ and $\mathrm{B}$ were lower than those in group $\mathrm{A}(P<0.05)$. This suggests that the clinical efficacy and safety of applying the herbal-scraping duplex method based on holographic theory for the treatment of CSD is ideal and has the value of promotion.
\end{abstract}

\section{Introduction}

Chronic subjective dizziness (CSD) is a group of chronic nonspecific syndromes with high sensitivity to motor signals and low tolerance to visual signals, accompanied by a significant subjective dizziness and instability [1]. Patients often seek medical attention for chronic and persistent head dizziness or a subjective sense of instability, which accounts for approximately $10.6 \%$ of all dizziness visits in outpatient clinics and can be twice as common in women as in men [2]. Psychiatric factors such as anxiety, depression, and panic are common triggers, and psychiatric medications are often applied symptomatically in clinical practice [3]. However, in view of the long treatment cycle of CSD, the safety and compliance of long-term use of such drugs are difficult to guarantee, and some patients have drug resistance or lack of efficacy [4]. Therefore, there is still a need to find a safer and more secure treatment method in the clinic.

CSD belongs to the category of "vertigo" in Chinese medicine. The patient is characterized by head dizziness, which may also be accompanied by complications related to dizziness. The onset is often related to the patient's emotional and mental disorders, improper diet, long-term illness and physical deficiency, old age and physical weakness, or 
fall injuries. Treatment can start by regulating the Qi of the whole body and taking into account the overall symptoms. In this study treatment, based on the holographic theory, herbal medicine, scraping, and music therapy, which are highly accepted by the patients, were used as treatment tools. Among them, the application of holographic soup is the result of concentrating the theory of holographic nutrition of herbs and the systemic therapy of Chinese medicine, which has the effect of overall regulation. The application of holographic scraping can achieve the purpose of adjusting the function of the corresponding viscera by stimulating the local information acupoint areas of the patient [5]. The application of five-element music, which combines the patient's condition and psychological state to dialectically play music for treatment, can achieve the effect of stretching the patient's Qi and emotions [6]. The application of all methods, through the combination of overall and local conditioning, is expected to achieve the physiological state of balance between Yin and Yang in CSD patients and ultimately help the body's function to recover as soon as possible and improve the condition. This study observes the clinical efficacy of the herbal-scraping duplex method in the treatment of CSD based on the holographic theory. The report is as follows.

\section{Materials and Methods}

2.1. Research Object. Between January 2020 and January 2021, 180 patients with CSD who met the inclusion criteria were randomly and equally divided into the group $\mathrm{A}$ $(n=60)$, the group $\mathrm{B}(n=60)$, and the group $\mathrm{C}(n=60)$. The general data difference analysis in Table 1 of the 3 groups was not statistically significant and could be used for experimental analysis $(P>0.05)$.

2.2. Diagnostic Criteria. Western medicine diagnostic criteria refer to the 2017 Bárány Society diagnostic criteria for CSD [7]: nonrotational dizziness or subjective imbalance of balance for $\geq 3$ months; high sensitivity to movement of things around or of themselves for $\geq 3$ months; dizziness worsens when performing refined visual tasks or in a complex visual environment. It might be accompanied by a sense of mental unclearness, or anxiety, depression, or somatization symptoms such as palpitations and pain. The diagnostic criteria of TCM referred to the diagnostic criteria of vertigo in the 2007 edition of "Traditional Chinese Medicine Encephalopathy." Main symptoms are obvious vertigo, a sense of shaking and spinning or vision rotation, with chronic onset and recurrent attacks. Secondary symptoms are nausea and vomiting, palpitation and irritability, drowsiness, insomnia and dreaminess, forgetfulness, tinnitus and deafness, fear and suspicion, and sweating and pallor.

2.3. Inclusion Criteria. Inclusion criteria were as follows: those with clear diagnostic criteria for CSD in Chinese and Western medicine; 30-70 years old; elementary school education or above, normal mental and cognitive ability, normal communication and understanding ability, and could cooperate to complete the relevant scale test; those without significant mental illness; those with normal neuroimaging; those with normal or mildly abnormal vestibular function and balance function tests, but not enough to make a diagnosis of abnormality; those who had not received other disease-related treatments within 3 months before treatment; and informed consent, voluntary participants.

2.4. Exclusion Criteria. Exclusion criteria were as follows: those whose dizziness was caused by other causes such as drugs, trauma, and poisoning; those who belong to other systemic disease-related dizziness, such as hypotension, hypoglycemia, anemia, hyper or hypothyroidism, and active neurootologic diseases; those with ophthalmoplegia, ocular muscle paralysis, leukemia, disorders of acid-base balance, electrolyte disorders, reduced cardiac ejection, and vascular diseases; those with cervical vertigo, peripheral vertigo, or central vertigo; persons with severe heart, brain, liver, and kidney dysfunction; the presence of a known drug dependency or alcoholism; those with unstable vital signs; pregnant and lactating women.

2.5. Rejection and Shedding Criteria. Rection and shedding criteria were as follows: those whose condition deteriorated rapidly due to serious complications or adverse reactions during the trial, those who developed other diseases that can cause vertigo during the trial, those who caused treatment suspension or the subject withdrew halfway through various factors, those whose medication did not meet the regulations or had low compliance with treatment, those who received other treatments that affect the efficacy during the observation period. Various factors led to loss of follow-up.

\subsection{Treatment Methods}

(1) Group A: received fluoxetine hydrochloride capsules (Suzhou Yushi Pharmaceutical Co., Ltd., Chinese Medicine Zhunzi H20093454) treatment, once a day, orally 30 minutes after breakfast, $20 \mathrm{mg}$ each time, continuous treatment for 4 weeks.

(2) Group B: on the basis of group A, holographic soup was added. Holographic soup recipe: $12 \mathrm{~g}$ each of Radix Bupleuri and Alisma orientale and $10 \mathrm{~g}$ each of Guizhi, Trichosanthes kirilowii, immature bitter orange, Allii Macrostemonis Bulbus, Magnolia officinalis cortex, Paeonia lactiflora Pall., Atractylodes sinensis, dried tangerine or orange peel, Atractylodes macrocephala, Poria cocos, Polyporus, Radix Rehmanniae, Cortex Moutan, ginger, jujube, and licorice. Addition and subtraction for clinical evidence: palpitations and irritability with $12 \mathrm{~g}$ each of keel and oyster; fatigue and sleepiness with $110 \mathrm{~g}$ of Rhizoma Acori Tatarinowii; tinnitus and deafness with $10 \mathrm{~g}$ each of Medulla Tetrapanacis and Rhizoma Acori 
TABLE 1: Comparison of the distribution of the 3 groups of general data.

\begin{tabular}{lccc}
\hline Group & Male/female $(n(\%))$ & Age $(\bar{x} \pm s$, years old $)$ & Course of disease $(\bar{x} \pm s$, years $)$ \\
\hline Group A $(n=60)$ & $18 / 42(30.00 / 70.00)$ & $49.25 \pm 4.64$ & $3.71 \pm 1.05$ \\
Group B $(n=60)$ & $21 / 39(35.00 / 65.00)$ & $48.63 \pm 5.14$ & $4.03 \pm 1.42$ \\
Group C $(n=60)$ & $19 / 41(31.67 / 68.33)$ & $48.71 \pm 4.97$ & $3.87 \pm 1.15$ \\
$\chi^{2} / F$ & 0.356 & 0.282 & 1.038 \\
$P$ & 0.837 & 0.755 & 0.357 \\
\hline
\end{tabular}

Tatarinowii. All herbal tablets were sourced from the Chinese pharmacy of our hospital. Each dose of Chinese medicine was decocted with $400 \mathrm{~mL}$ of water routinely, and patients were instructed to take $200 \mathrm{~mL}$ each in the morning and evening after adding warmth. 1 week was 1 course of treatment, continue treatment for 4 courses.

(3) Group C: on the basis of group B, holographic scraping therapy was added. Holographic scraping and acupoint selection: on Monday, the holographic acupoint area took the middle of the forehead of the head, 1 area of the right side of the forehead, and 2 areas of the left side of the forehead, head, and neck meridian acupoints took the odd points, Sishencong, blood pressure points. On Tuesday, the holographic acupoint area took the posterior $1 / 3$ of the forehead parietal zone and the lower $1 / 3$ of the parietal posterior oblique zone; meridian acupoints took the Baihui-Fengfu of the governor vessel. On Wednesday, the holographic acupoint area took the spleen and stomach correspondence area of the spine, and the meridian acupoints took the Pishu, Weishu, Xinshu, and Shenshu of the bilateral bladder meridian. On Thursday, the holographic acupoint area took the head acupoints and heart acupoints on the radial side of the second metacarpal bone of the hand and palm middle finger, and the meridian acupoints took the Shenmen of the bilateral heart meridian. On Friday, the holographic acupoint area took the hypotensive ditch on the back of the ear, and the meridian acupoints took the Zusanli of bilateral stomach meridian, the Sanyinjiao of bilateral spleen meridian, the Yongquan of bilateral kidney meridian. Operation: before scraping, examine the patient's local skin for ulcers and breaks and ask the patient about contraindications to scraping. When scraping, guide the patient to a comfortable posture and fully expose the scraping area. After routine disinfection, use a flat replenishing-reducing technique for scraping at a speed of 60 times/min. During the period, pay close attention to the patient's local reaction and tolerance. Do not use excessive force and take comfort as the standard, once a day from Monday to Friday, every 20-30 minutes. During each Gua Sha, the five-element music should be played dialectically based on the patient's condition and psychological state. It contains five corresponding pieces of Jiao, Zheng, Gong, Shang, and Yu (all created by the China Medical Audiovisual Publishing House). When playing music, it was necessary to ensure that the room is not disturbed by other noises or noises, and the playback volume must be controlled below 60 decibels. 1 week was 1 course of treatment; continue treatment for 4 courses.

\subsection{Observation Indicators}

(1) Dizziness handicap inventory (DHI) score: before and after treatment, the total scores of physical, social function, and emotion factors in the 3 groups of DHI were counted, with a total score of $0-100$ points. The higher the score, the more severe the dizziness handicap.

(2) TCM symptoms score: before and after treatment, the total scores of TCM syndromes of the 3 groups, such as dizziness, nausea and vomiting, palpitations, irritability, drowsiness, insomnia, forgetfulness, tinnitus, and deafness, were counted. The scores $(0,2,4$, and 6$)$ are proportional to the severity of the syndrome (none, mild, moderate, and severe).

(3) Hamilton anxiety/depression scale (HAMA/ HAMD) score: before and after treatment, HAMA/ HAMD assessed the degree of anxiety and depression in the 3 groups. The former with a total score of 0-56 points; the latter with a total score of 0-68 points. The higher the score, the greater the anxiety and depression.

(4) Pittsburgh sleep quality index (SQI) score: before and after treatment, PSQI assessed the total scores of the 3 groups of factors such as sleep quality, sleep time, and sleep latency. The total score is $0-21$ points; the higher the score, the more severe the sleep disorder.

(5) Fatigue severity scale (FSS) score: before and after treatment, FSS assessed the fatigue degree of the 3 groups and their impact on daily functions, with a total score of 9-63 points. The higher the score, the more severe the fatigue.

(6) Others: during the treatment, the 3 groups of patients were subjected to blood routine, urine routine, stool routine, liver and kidney function, and electrocardiogram and other safety checks, and the occurrence of adverse of the 3 groups was observed such as nausea, vomiting, constipation, skin rash, dry mouth, and so on. After 1 month of drug discontinuation, the recurrence was followed up and counted in the 3 groups. 


\subsection{Efficacy Evaluation}

(1) Western medicine efficacy determination criteria: formulated according to the improvement of the DHI score. (1) Healed: efficacy index $\geq 75 \%$. (2) Excellent: $75 \%>$ efficacy index $\geq 50 \%$. (3) Improved: $50 \%>$ efficacy index $\geq 25 \%$. (4) Ineffective: efficacy index $<25 \%$. Efficacy index $=$ (before treatment - after treatment)/before treatment score $\times 100 \%$. Total effective $=($ healed + excellent + improved $)$ number.

(2) Chinese medicine efficacy determination criteria: formulated according to the 2012 edition of the "Diagnosis and Efficacy Criteria for Diseases and Syndromes of Traditional Chinese Medicine." (1) Healed: efficacy index $\geq 95 \%$. (2) Excellent: 95\% $>$ efficacy index $\geq 70 \%$. (3) Improved: $70 \%>$ efficacy index $\geq 30 \%$. (4) Ineffective: efficacy index $<30 \%$. Efficacy index $=$ (before treatment-after treatment $) /$ before treatment score $\times 100 \%$. Total effective $=$ (healed + excellent + improved) number.

2.9. Statistical Methods. SPSS 22.0 software was applied. The test level was $P=0.05$. Normally distributed measures were expressed as $(\bar{x} \pm s)$, one-way ANOVA was performed for comparisons between multiple groups, and the $t$-test was performed for two-way comparisons between groups. Count data were expressed as (\%), and $\chi^{2}$ was performed. Statistical differences between the data were indicated at $P<0.05$.

\section{Results}

3.1. Comparison of 3 Groups of DHI and TCM Scores. After treatment, the total scores of DHI and TCM in the 3 groups were lower than before, and the scores of group $\mathrm{C}$ were lower than those of groups B and A, and group B was lower than group A $(P<0.05)$ (Figures 1 and 2 ).

3.2. Comparison of 3 Groups of DHI and TCM Scores Treatment Efficiency. After treatment, in the total effective rate of the DHI score, group C $(95.00 \%)>$ group B $(86.67 \%)>$ group A (71.67\%); in the total effective rate of the TCM score, group C $(88.33 \%)>$ group B $(81.67 \%)>$ group A (65.00\%). After treatment, the total effective rates of DHI and TCM scores of group $\mathrm{C}$ and group $\mathrm{B}$ were statistically different from those of group A $(P<0.05)$, and there was no statistical difference between group $\mathrm{C}$ and group $\mathrm{B}$ $(P>0.05)$ (Tables 2 and 3$)$.

3.3. Comparison of 3 Groups of HAMA and HAMD Scores. After treatment, the scores of HAMA and HAMD in the 3 groups were lower than before, and the scores of group $\mathrm{C}$ were lower than those of groups $\mathrm{B}$ and $\mathrm{A}$, and group $\mathrm{B}$ was lower than group A $(P<0.05)$ (Figures 3 and 4 ).

3.4. Comparison of 3 Groups of PSQI Scores. After treatment, the PSQI scores of the 3 groups were lower than those before, and the scores of group $\mathrm{C}$ were lower than those of groups $\mathrm{B}$

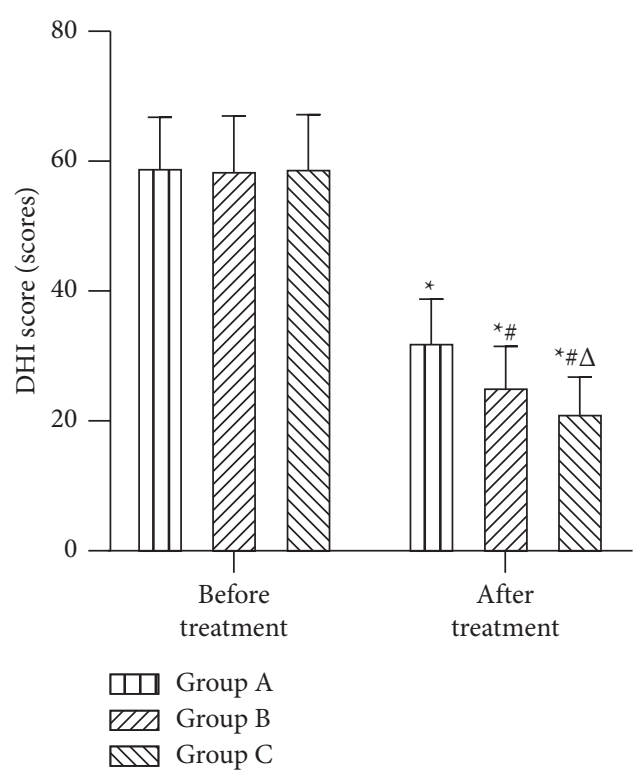

Figure 1: Comparison of 3 groups of DHI scores $(\bar{x} \pm s$, scores). Compared with the same group before treatment, ${ }^{*} P<0.05$; compared with group A after treatment, ${ }^{\#} P<0.05$; compared with group B after treatment, ${ }^{\Delta} P<0.05$.

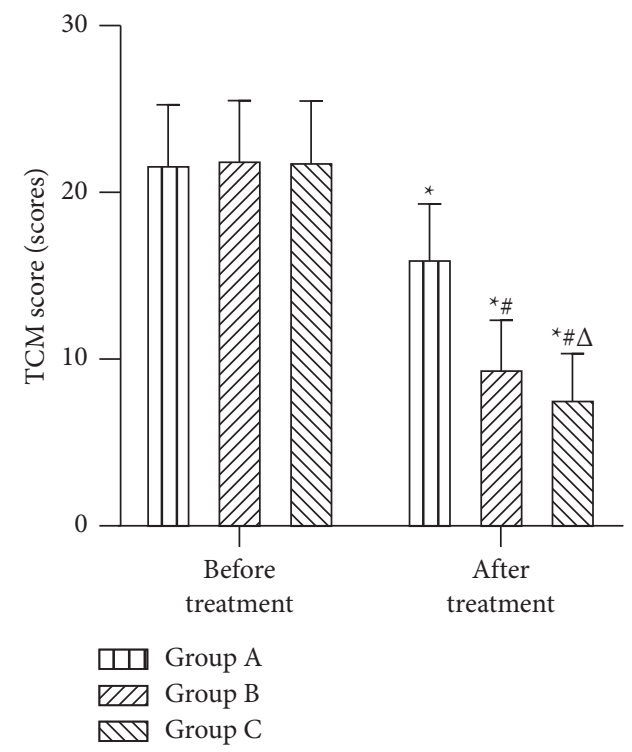

Figure 2: Comparison of 3 groups of TCM scores ( $\bar{x} \pm s$, scores). Compared with the same group before treatment, ${ }^{*} P<0.05$; compared with group A after treatment, ${ }^{\#} P<0.05$; compared with group B after treatment, ${ }^{\Delta} P<0.05$.

and $\mathrm{A}$, and group $\mathrm{B}$ was lower than group $\mathrm{A}(P<0.05)$ (Figure 5).

3.5. Comparison of 3 Groups of FSS Scores. After treatment, the FSS scores of the three groups were lower than before, and the scores of group $\mathrm{C}$ were lower than those of group $\mathrm{B}$ and $\mathrm{A}$, and group $\mathrm{B}$ was lower than group $\mathrm{A}(P<0.05)$ (Figure 6). 
TABLE 2: Comparison of 3 groups of DHI score treatment efficiency $(n(\%))$.

\begin{tabular}{lccccc}
\hline Group & Healed & Excellent & Improved & Ineffective & Total effective rate $(\%)$ \\
\hline Group A $(n=60)$ & 2 & 16 & 25 & 17 & $43(71.67)$ \\
Group B $(n=60)$ & 6 & 29 & 17 & 8 & $52(86.67)^{*}$ \\
Group C $(n=60)$ & 8 & 36 & 13 & 3 & $57(95.00)^{*}$ \\
$\chi^{2}$ & & & & 12.773 \\
$P$ & & & & 0.002 \\
\hline
\end{tabular}

Compared with group $\mathrm{A},{ }^{*} \mathrm{P}<0.05$.

TABLE 3: Comparison of 3 groups of TCM score treatment efficiency ( $n(\%)$ ).

\begin{tabular}{lccccc}
\hline Group & Healed & Excellent & Improved & Ineffective & Total effective rate $(\%)$ \\
\hline Group A $(n=60)$ & 1 & 10 & 28 & 21 & $39(65.00)$ \\
Group B $(n=60)$ & 4 & 25 & 20 & 11 & $49(81.67)^{*}$ \\
Group C $(n=60)$ & 5 & 30 & 18 & 7 & $53(88.33)^{*}$ \\
$\chi^{2}$ & & & & 10.213 \\
$P$ & & & & 0.006 \\
\hline
\end{tabular}

Compared with group $\mathrm{A},{ }^{*} \mathrm{P}<0.05$.

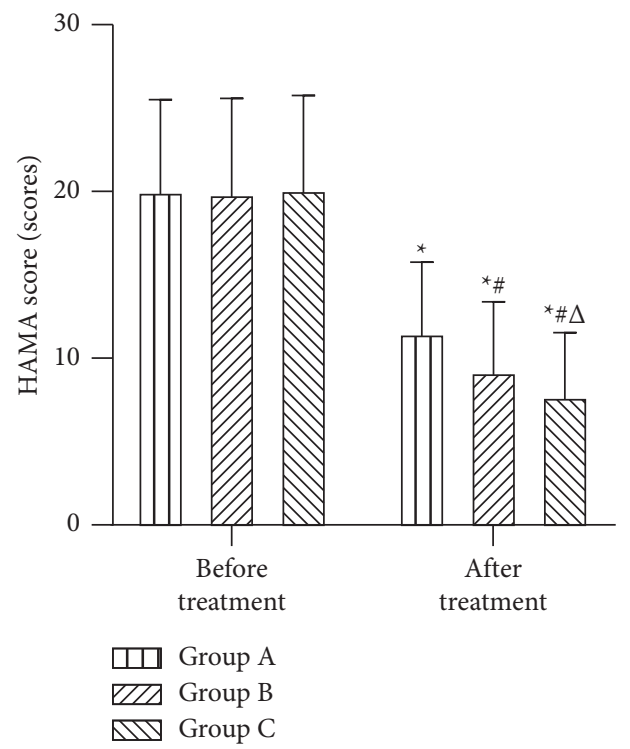

FIGURE 3: Comparison of 3 groups of HAMA scores $(\bar{x} \pm s$, scores). Compared with the same group before treatment, ${ }^{*} P<0.05$; compared with group A after treatment, ${ }^{\#} P<0.05$; compared with group B after treatment, ${ }^{\Delta} P<0.05$.

3.6. Comparison of 3 Groups of the Adverse Reaction Rate and Recurrence Rate. During the treatment period, 2 patients in group A developed constipation and 2 patients with nausea and retching, with an adverse reaction rate of $6.67 \%$; 2 patients in group B had diarrhea and 1 patient with nausea, with an adverse reaction rate of $5.00 \%$; patients in group $\mathrm{C}$ had 1 case of diarrhea, 1 case of headache, and 1 case of dry mouth, and the adverse reaction rate was $5.00 \%$. There was no statistical difference in the adverse reaction rate of the 3 groups $(P>0.05)$. One month after drug withdrawal, 17 cases in group A recurred, with a recurrence rate of $28.33 \% ; 8$ cases in group $\mathrm{B}$ recurred, with a recurrence rate of $13.33 \%$; and 6 cases in group $\mathrm{C}$ recurred, with a recurrence rate of $10.00 \%$. The recurrence rates of group $\mathrm{C}$ and group $\mathrm{B}$ were statistically

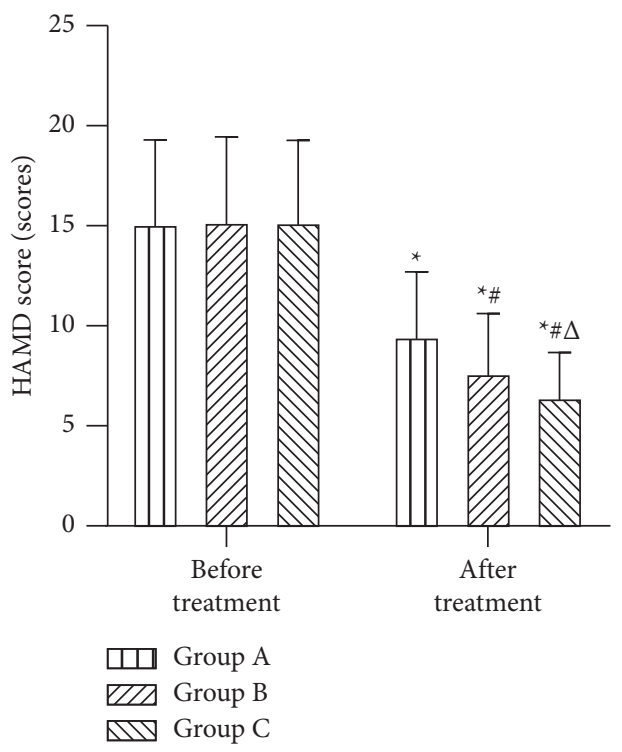

FIGURE 4: Comparison of 3 groups of HAMD scores ( $\bar{x} \pm s$, scores). Compared with the same group before treatment, ${ }^{*} P<0.05$; compared with group A after treatment, ${ }^{\#} P<0.05$; compared with group B after treatment, ${ }^{\Delta} P<0.05$.

different from group A $(P<0.05)$, and there was no statistical difference between group $\mathrm{C}$ and group $\mathrm{B}(P>0.05)$ (Figure 7).

\section{Discussion}

CSD was first called "space motion discomfort," "phobic positional vertigo," and "visual vertigo" [8]. All 3 described a phenomenon in which patients have strong discomfort or hypersensitivity to spatial position signals or complex visual signals. In addition, it has been reported that $75 \%$ of patients with phobic positional vertigo can be accompanied by significant symptoms of anxiety and depression, and it is also common in patients with visual vertigo [9]. Therefore, chronic dizziness without clear vestibular dysfunction has been called 


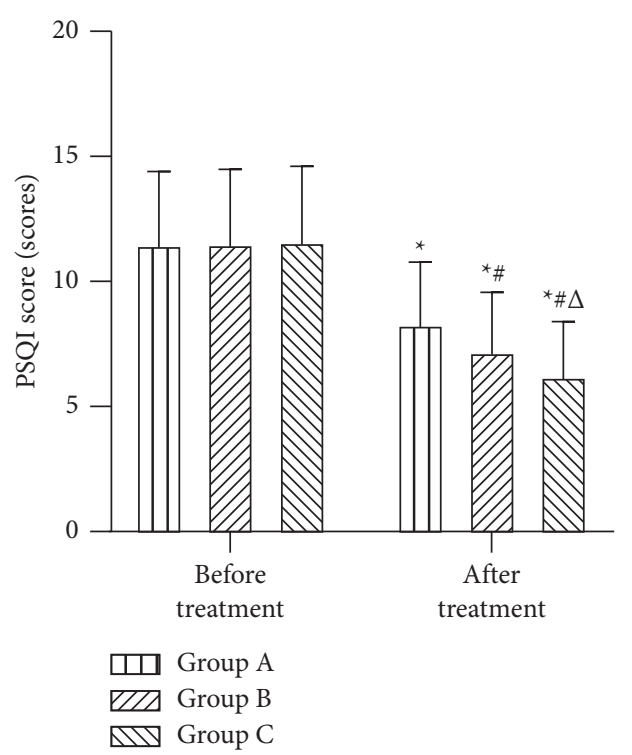

FIGURE 5: Comparison of 3 groups of PSQI scores $(\bar{x} \pm s$, scores). Compared with the same group before treatment, ${ }^{*} P<0.05$; compared with group A after treatment, ${ }^{\#} P<0.05$; compared with group B after treatment, ${ }^{\Delta} P<0.05$.

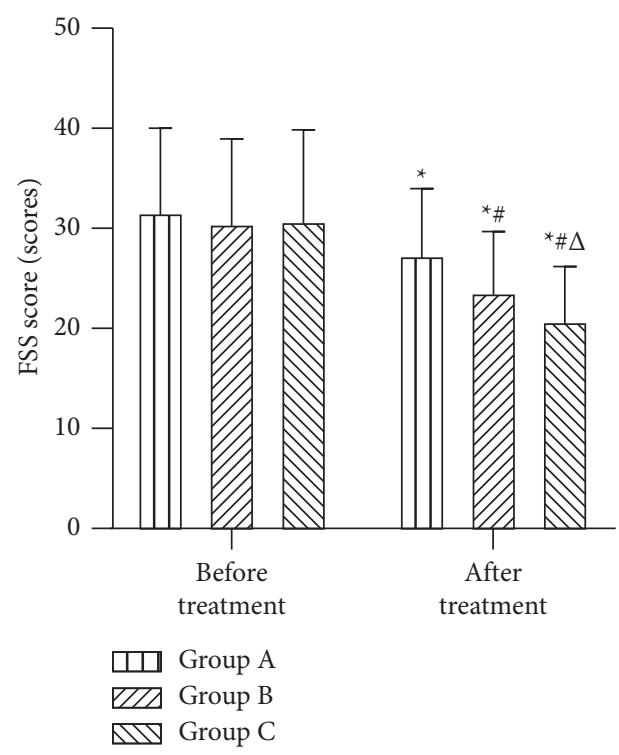

FIGURE 6: Comparison of 3 groups of FSS scores $(\bar{x} \pm s$, scores). Compared with the same group before treatment, ${ }^{*} P<0.05$; compared with group A after treatment, ${ }^{\#} P<0.05$; compared with group B after treatment, ${ }^{\Delta} P<0.05$.

“mental dizziness" for a long time. From 2004 to 2005, Staab and Ruckenstein integrated the above concepts and proposed the name of CSD [10]. They believe that physical diseases (such as past neurological and otological diseases) or mental diseases (such as anxiety, depressive personality) are the main predisposing factors for CSD [11]. The clinical symptoms of the patients will not disappear with the disappearance of these predisposing factors, but will show a lingering trend with the varying degree of the patient's condition. Based on the above, the clinical use of selective serotonin reuptake inhibitor (such as

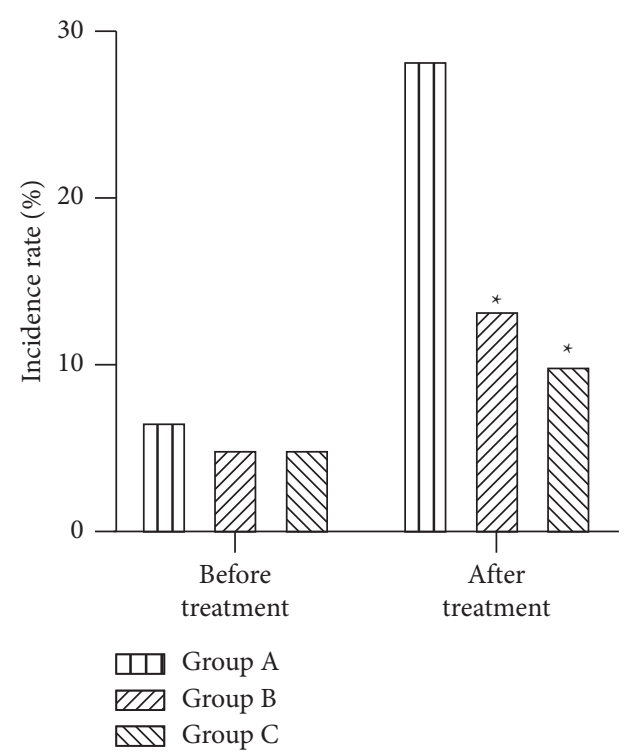

FIgURE 7: Comparison of 3 groups of the adverse reaction rate and recurrence rate $(n(\%))$. Compared with group $\mathrm{A},{ }^{*} P<0.05$.

fluoxetine, paroxetine, and sertraline) for the treatment of mental and psychological disorders can significantly improve or even completely relieve the clinical symptoms of some patients, but some patients still experience drug resistance reactions or drug side effects in long-term applications or discontinuation reactions such as palpitations, irritability, dizziness, and weakness after discontinuation of the drug [12, 13]. More safe and effective treatments still need to be sought clinically.

Through long-term clinical practice in Chinese medicine, the author found that in addition to the common symptoms of dizziness, patients with CSD may also have a series of symptoms of autonomic dysfunction such as anxiety, depression, insomnia, forgetfulness, tinnitus, lethargy, drowsiness, and dullness. Therefore, the treatment should focus on the whole, with the principle of regulating Yin and Yang, mediating the Qi machine, and dredging the surface, interior, upper, middle, and lower. Based on this, the application of holographic soup in this study is based on the theory of holographic nutrition of Chinese herbs, which believes that a certain material form or nutritional sum in Chinese herbs has the effect of preventing and controlling a corresponding certain disease. It is believed that a certain substance form or total nutrition in Chinese medicinal materials has the effect of preventing and curing corresponding diseases. This formula combines decoction of immature bitter orange, minor bupleurum decoction, cinnamon twig decoction, peptic powder, and Wuling powder in one formula. Among them, decoction of immature bitter orange can dredge Yang and dispel stagnation, resolve phlegm, and promote Qi and treat the syndrome of stagnation of phlegm and Qi in the upper heater [14]. The application of the core herbs Radix Bupleuri and licorice in minor bupleurum decoction can promote Yang and regulate Qi, harmonize Shaoyang, and mediate the gas machine in the middle heater [15]; Cinnamon twig decoction can evacuate the wind chill, reconcile the nutrient and defense, 
and clear the head and eyes. Peptic powder can reduce dampness and invigorate spleen, regulate Qi and stomach, and treat the dampness and Qi resistance in the middle heater [16]. The application of Wuling powder can invigorate spleen and dispel dampness, promote Yang and diuresis, and treat the symptom of stagnation of water in the lower heater [17]. In addition, Shengdi and Moutan bark can be used to cool blood and disperse blood stasis, which can treat the syndrome of the heat-stasis in blood. The herbs are combined to unify and regulate triple burner, which has the overall regulating effect of unblocking the surface, interior, upper, middle, and lower, and communicating nutrient, defense, Qi, and blood.

In addition, the theory of TCM believes that the human body is an organic unity, and the physiological activities or pathological changes of any local organs can affect the overall physiological and pathological responses. The dysfunction of the overall function will inevitably lead to the physiological and pathological changes of the local organs. By extension, the phenomenon that a relatively independent part of an organism contains all the information of the whole is a universal law, and this is the holographic law of living things [18]. Based on this theory, the ancients believed that the concepts of the five viscera, six internal organs, Qi and blood, and pulse signs are all holographic embryo or external manifestations of the holographic embryo. They also applied local therapies such as head acupuncture, ear acupuncture, wrist-ankle acupuncture, and foot therapy to regulate the overall functional state of the patient and by stimulating the projections of the body's five internal organs and six internal organs in the corresponding acupuncture areas of the head, hands, and feet to achieve the effect of disease prevention and treatment, which is known as holographic therapy $[19,20]$. Based on the above recognition, this study applied holographic scraping and five-element music to CSD patients in group C. The results showed that after treatment, the scores of DHI, TCM, HAMA, HAMD, PSQI, and FSS in the 3 groups were lower than those before, and the scores of group $C$ were lower than those of group $B$ and group $A$, and group B was lower than group A $(P<0.05)$. After treatment, the total effective rates of DHI and TCM scores of group C and group $B$ were statistically different from those of group A $(P<0.05)$, and there was no statistical difference between group $\mathrm{C}$ and group $\mathrm{B}(P>0.05)$. This suggests that based on holographic theory, the herbal-scraping duplex method treatment is more effective in improving vertigo symptoms, mental symptoms, sleep disorders, and fatigue symptoms in CSD patients than simple drug treatment. This group of holographic scraping is a type of scraping therapy that promotes blood circulation and removes blood stasis or detoxification to the patient's local area, regulates the corresponding viscera functions, promotes the balance of Yin and Yang, and improves physical fitness. In previous studies, after holographic scraping, the body's antiviral ability and gastrointestinal absorption function of patients with headache after mixed hemorrhoid surgery were significantly improved. In this group of holographic scraping, by appropriately stimulating the patient's holographic and meridian acupoints, it can promote the decomposition of some tissue cell proteins and the production of histamine or histamine-like and can expand local capillaries and promote blood and lymph microcycle. As a result, the hypoxic state and material metabolism of the patient's local tissues can be improved, which not only helps to achieve the effect of disease prevention and treatment but also can play the role of adjusting the whole by local holographic conditioning; ultimately, patients can fully mobilize their own potential and promote the improvement of disease susceptibility. The coordinated application of the five-element music, combined with patient's illness and mental state dialectically play the corresponding music during holographic scraping, can promote the patient's peace of mind and comfort, and help relieve their anxiety, depression, and other mental symptoms [21]. Therefore, it also has the effect of improving subjective dizziness.

The results of this study also showed that during the treatment period, there was no statistical difference in the adverse reaction rate of the 3 groups $(P>0.05)$. This suggests that the safety of the application of the herbal-scraping duplex method to CSD based on the holographic theory can be guaranteed. In this study, the recurrence rate of the 3 groups was observed after 1 month of drug discontinuation, and the results showed that the recurrence rate of group $\mathrm{C}$ was $10.00 \%<13.33 \%$ in group $B<28.33 \%$ in group $A$. The recurrence rates of group $C$ and group $B$ were statistically different from group A $(P<0.05)$, and there was no statistical difference between group $\mathrm{C}$ and group $\mathrm{B}(P>0.05)$. This suggests that integrated traditional Chinese and Western medicine is more effective than simple Western medicine in improving the recurrence rate of patients, and it has a promotion value.

\section{Conclusion}

In conclusion, this study based on the holographic theory of the herbal-scraping duplex method applied to CSD patients had better results in improving symptoms and accelerating healing, and safety was ensured. In terms of reducing shortterm recurrence, the two integrated traditional Chinese and Western medicine treatments are better than simple Western medicine treatment, but in the two integrated traditional Chinese and Western medicine treatments, the combined application of the herbal-scraping duplex method has no significant superimposed effect, and the observation of their long-term efficacy and recurrence still needs further in-depth study in the future.

\section{Data Availability}

The primary data used to support the results of this study are available from the corresponding author upon request.

\section{Ethical Approval}

This study was approved by the ethics committee of our hospital. 


\section{Disclosure}

Huanwen Luo and Caidan Liu are the co-first authors.

\section{Conflicts of Interest}

The authors declare that they have no conflicts of interest.

\section{References}

[1] M. Baydan, O. Yigit, and S. Aksoy, "Does vestibular rehabilitation improve postural control of subjects with chronic subjective dizziness?" PLoS One, vol. 15, no. 9, Article ID e0238436, 2020.

[2] M. Ödman and R. Maire, "Chronic subjective dizziness," Acta Oto-Laryngologica, vol. 128, no. 10, pp. 1085-1088, 2008.

[3] X. Chen, J. Cheng, and J. Gong, "Deanxit can improve the dizziness, anxiety, and quality of life of patients with chronic subjective dizziness," American Journal of Tourism Research, vol. 13, no. 8, pp. 9348-9355, 2021.

[4] D. Lochmann and T. Richardson, "Selective serotonin reuptake inhibitors," Antidepressants, vol. 250, pp. 135-144, 2019.

[5] L. Li, P. Zhang, Z. Qin et al., "The effect of holographic meridian scraping therapy combined with free position on the labor process, perineum lateral resection rate, and delivery outcomes of primiparae," American Journal of Tourism Research, vol. 13, no. 8, pp. 9846-9852, 2021.

[6] Q. Wu, Z. Liu, X. Pang, and L. Cheng, "Efficacy of five-element music interventions in perinatal mental health and labor pain: a meta-analysis," Complementary Therapies in Clinical Practice, vol. 40, Article ID 101217, 2020.

[7] J. P. Staab, A. Eckhardt-Henn, A. Horii et al., "Diagnostic criteria for persistent postural-perceptual dizziness (PPPD): consensus document of the committee for the Classification of Vestibular Disorders of the Bárány Society," Journal of Vestibular Research, vol. 27, no. 4, pp. 191-208, 2017.

[8] J. P. Staab, A. Eckhardt-Henn, A. Horii et al., "Diagnostic criteria for persistent postural-perceptual dizziness (PPPD): consensus document of the committee for the Classification of Vestibular Disorders of the Bárány Society," Journal of Vestibular Research, vol. 27, no. 4, pp. 191-208, 2017.

[9] J. Huber, V. L. Flanagin, P. Popp, P. Eulenburg, and M. Dieterich, "Network changes in patients with phobic postural vertigo," Brain and Behavior, vol. 10, no. 6, Article ID e01622, 2020.

[10] D. L. McCaslin, G. P. Jacobson, H. L. Burrows, P. Littlefield, and D. S. Haynes, "Transforming superior canal dehiscence to chronic subjective dizziness: from SCD to CSD," Journal of the American Academy of Audiology, vol. 21, no. 05, pp. 293-300, 2010.

[11] J. P. Staab and M. J. Ruckenstein, "Chronic dizziness and anxiety," Archives of Otolaryngology - Head and Neck Surgery, vol. 131, no. 8, pp. 675-679, 2005.

[12] A. Horii, T. Imai, T. Kitahara et al., "Psychiatric comorbidities and use of milnacipran in patients with chronic dizziness," Journal of Vestibular Research, vol. 26, no. 3, pp. 335-340, 2016.

[13] B. Bandelow, "Current and novel psychopharmacological drugs for anxiety disorders," Advances in Experimental Medicine \& Biology, vol. 1191, pp. 347-365, 2020.

[14] Y. Tang, H. Cai, Z. Zhan et al., "Herbal medicine (zhishi xiebai guizhi decoction) for unstable angina," Medicine, vol. 97, no. 52, p. e13965, 2018.
[15] S. Shao, R. Jia, L. Zhao et al., "Xiao-Chai-Hu-Tang ameliorates tumor growth in cancer comorbid depressive symptoms via modulating gut microbiota-mediated TLR4/MyD88/NF- $\kappa \mathrm{B}$ signaling pathway," Phytomedicine, vol. 88, Article ID 153606, 2021.

[16] S. P. Hu, "Recurrent respiratory infection in children treated with assistant of Ping-Wei powders--report of 45 cases," Zhongguo Zhong Xi Yi Jie He Za Zhi, vol. 28, pp. 85-86, 2008.

[17] H.-N. Zhou, H.-Y. Li, W.-H. Xu et al., "Study on the action mechanism of Wuling Powder on treating osteoporosis based on network pharmacology," Chinese Journal of Natural Medicines, vol. 19, no. 1, pp. 28-35, 2021.

[18] Y. Luo, M. Yang, T. Liu et al., "Effect of hand-ear acupuncture on chronic low-back pain: a randomized controlled trial," Journal of traditional Chinese medicine $=$ Chung $i$ tsa chih ying wen pan, vol. 39, pp. 587-598, 2019.

[19] Z. L. Wang, L. F. Chen, and W. M. Zhu, "Observation on the transient analgesic effect of abdominal acupuncture TENS on pain of neck, shoulder, loin and legs," Zhongguo Zhen Jiu, vol. 27, pp. 657-659, 2007.

[20] Y. You, T. Zhang, S. Shu, X. Qian, S. Zhou, and F. Yao, "Wristankle acupuncture and Fluoxetine in the treatment of poststroke depression: a randomized controlled clinical trial," Journal of traditional Chinese medicine, vol. 40, pp. 455-460, 2020.

[21] J. Liao, Y. Wu, Y. Zhao et al., "Progressive muscle relaxation combined with Chinese medicine five-element music on depression for cancer patients: a randomized controlled trial," Chinese Journal of Integrative Medicine, vol. 24, no. 5, pp. 343-347, 2018. 